(C2007 IEEE. Personal use of this material is permitted. However, permission to reprint/republish this material for advertising or promotional purposes or for creating new collective works for resale or redistribution to servers or lists, or to reuse any copyrighted component of this work in other works must be obtained from the IEEE. 


\title{
Exploring a Digital Ecosystem Conceptual Model and Its Simulation Prototype
}

\author{
Chen Wu, Elizabeth Chang \\ Digital Ecosystems and Business Intelligence Institute, Curtin University of Technology, Perth 6845 Australia \\ E-mail: \{Chen.Wu, Elizabeth.Chang\}@cbs.curtin.edu.au
}

\begin{abstract}
Digital Ecosystems (DES) transcend the traditional rigorously defined collaborative environments from centralised or distributed or hybrid models into an open, flexible, domain cluster, demand-driven interactive environment. A digital ecosystem is new-networked architecture and collaborative environment that addresses the weakness of client-server, peer-topeer and web services. In this paper, we firstly introduce a conceptual model of DES. The design and implementation of a DES simulation is then presented to illustrate some fundamental characteristics for further DES study. Finally, we propose a novel approach to realise the DES built on top of the Social Software (SS) and Swarm Intelligence.
\end{abstract}

\section{INTRODUCTION}

A digital ecosystem (DES) is a new networked architecture and collaborative environment that addresses the weakness of client-server, peer-to-peer and web services environments. In our previous work [1], we define a digital ecosystem as a "Loosely-coupled, demand-driven, domain clustered, agentbased collaborative environment where each species is proactive and responsive for its own benefit or profit". In order to foster such an open environment in our digital world, explorative simulations and prototypes can lead to a desirable vision for further research and implementation. We aim to provide a bottom-up approach to build a DES environment from an empirical perspective. In this paper, the design, implementation, and a demonstration of a DES simulation are presented to illustrate some fundamental characteristics of DES. Meanwhile, a preliminary realisation of DES concepts and simulation is provided as a DES collaboration platform built on top of MediaWiki [2] - the underlying software infrastructure for Wikipedia [3].

The rest part of this paper is organised as follows. Section II presents the conceptual model of DES. Section III proposes the conceptual model of the DES simulation system and its implementation. This is followed by the system demonstration presented in Section IV. In Section V, we provide a novel solution to address the realisation of DES - the social software approach. The paper concludes in Section VI.

\section{PRELIMINARY CONCEPTS}

In this section, we would like to elaborate some of the core concepts in the definition of DES for facilitating the understanding of the requirements to build a DES simulation prototype.

In an ecological system environment, we consider species analogous to biological species creating and conserving resources that humans find valuable. The software, databases, applications or software services in digital ecosystems are referred to as digital species.

We define loosely-coupled as a freely bound open relationship between participants or entities within a virtual community. This term is opposite to the tightly coupled relationship, where each party is heavily dependent on one another and the roles are pre-defined. Participant is defined as an entity who wants to join a group or an environment or a community based on its own interest.

We define open as a free transparent environment, where everyone is invited to join except dangerous species that have the intention of causing damage to the community.

We define flexible as an easy, tolerable and adaptable environment, with strength to survive peacefully. For example, if coral polyps die, they become a stony, branching structure as part of a reef and can still provide shelter generation after generation.

We define domain clustered as a colony or a field where participants have something in common or share the same life or interests, such as ocean habitats in coral reef or exotic tropical plants in a rainforest ecosystem.

Demand driven is defined as the driving force coming from outside 'push-in' rather than 'pull-in'. For example, the current networked economy has led supply chains to demand chain, where demand is volatile and supply is uncertain. Another negative example is that the current collaborative environment is not a demand driven environment because humans are told to collaborate and humans may be forced to collaborate. This is not demand driven and a human is forced to be there for the sake of collaboration rather than enjoying collaboration arising from a perceived mutual interest of the parties collaborating. There is no real honest consideration about whether there will be a benefit or profit from the collaboration to the collaborating parties.

Collaborative environment is defined as an environment, which contains human individuals, information technologies that humans can capitalise on; tools that facilitate interaction and knowledge sharing along with resources that help maintain synergy among human beings or software agents. 
Human agents and software agents in a digital ecosystem are referred to as eco-agents. Eco-agents are capable of acting autonomously; often capable of decision-making and responses within the context of a digital ecosystem.

Proactive is defined as an agent or eco-agent that is full of enthusiasm to participate in team work or community work. Responsive signifies an agent or eco-agent that demonstrates willingness, is passionate about the issues, is cooperative and takes responsibility for its action. Benefit refers to an advantage that an agent can take without any risks. Profit refers to personal gain (e.g. financial reward).

A digital ecosystem is a self-organising digital infrastructure aimed at creating a digital environment for networked organisations or agents that support cooperation, knowledge sharing and development of open and adaptive technologies and evolutionary domain knowledge rich environments through dynamic interactions within and between different domain clusters and their environment and collaborative effort to remain balanced in the ecosystem environment. It is a business model innovation in the digital economy.

\section{CONCEPTUAL MODEL}

In this section, we provide the conceptual model for DES simulation prototype. The conceptual model is inspired by the Swarm Simulation API [4] as shown in Fig 1.

At the top level, there are five types of conceptual components: Species, Scheduler, Activities, Rules, and the Space. We explain them in detail at the following sub-sections.

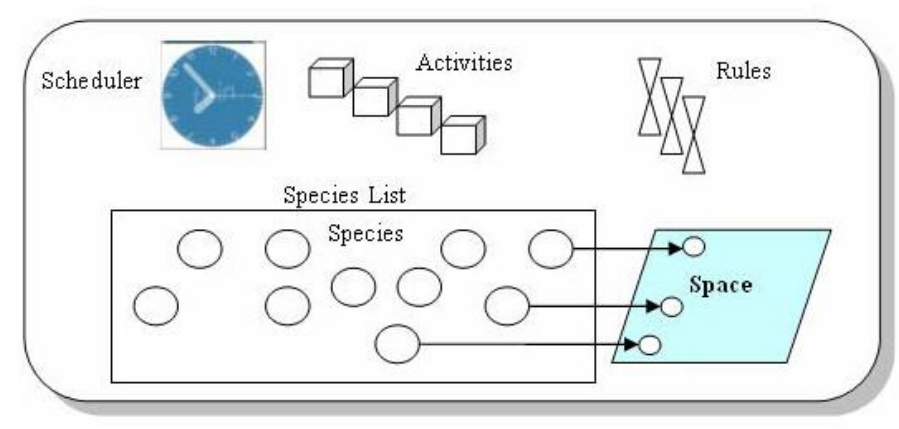

Figure 1. Conceptual Model

\section{A. Species}

Species are the primary components for the DES simulation. They are wandering around within the space. They follow certain rules to perform some predefined activities. Although each specimen has its own simple rules to follow, the collective behaviour sometimes could be quite surprising.

To manage these species, in our simulation, we register each specimen in a species list, where each specimen can be referenced. By doing so, we can send messages to all species. Therefore, they can observe what is going on within the digital ecosystem in a timely manner. Each specimen is also 'projected' into a space, which can be a physical two dimensional coordinate, a virtual multi-dimensional space, or a concrete network topology such as client-server, peer-to-peer network architecture. At the simulation level, we do not define the technical detail of such a projection. Rather, we leave this job to DES realisation implementation, where the detailed projection technology has to be instantiated.

There are basically two types of species: moving species and business opportunities. Although opportunities are not moving, we treat them as a kind of species for design consistency.

\section{B. Scheduler}

Scheduler is a special component that arranges the activities carried out by species. The simulation users interact with the scheduler at the design phase, i.e. setting certain parameters to the scheduler, which then regulates the 'timetable' for each specimen. Technically, the scheduler is activity-oriented; hence it is, in effect, independent of species.

\section{Activities}

If a scheduler sets the 'time-table' for each specimen, activities set the "to-do list" for each specimen. The activity stipulates the tasks that species have to perform. From the implementation angle, this could be a message sent to the species, which then respond to the message according to the rule set in its mind.

\section{Rules}

Rules are crucial for species. Without rules, species cannot 'think' and 'respond'. Rules represent the intelligence and the experiences that a species might processes. A rule is always associated with several activities. Put it simply, we can consider a rule as a black box unit, where the input is what species have encountered from its neighbouring milieu, and the output could be a set of activities. Thus rules help species to decide 'what to do under certain situations'. In current DES simulations, rules are explicitly set in UI by end users. In the future version of this simulation, we are looking for the approach to declare and embed rules into the species directly.

\section{E. Space}

In reality, Space is the social contexts where species live, interact, survive, prosper, or fade away. In this simulation, space can be a two dimensional coordinate, a virtual multidimensional space, or a concrete network topology such as client-server, peer-to-peer network architecture. Space set the boundary of the scope of species, thus activities conducted by the species have to stay in the boundary of certain Space defined or chosen by the simulation end users.

\section{F. Implementation}

We have used the Java Applet to implement this conceptual model. The class package is illustrated in Figure 2. From the class diagram, we can see that the class Canvas is the implementation of the conceptual component Space. The open environment is the DesimApplet, which contains the space. For the convenience, we have embedded the scheduler into the Canvas, and activities as the methods for the Class species. 


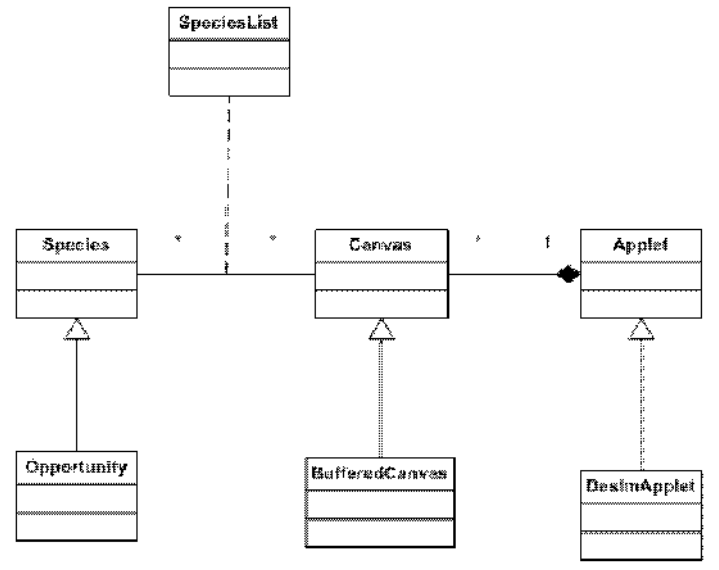

Figure 2 Class Diagram of DES Simulation prototype

\section{DES SIMULATION PROTOTYPE}

In this section, we provide the run-time system behaviour of our DES simulation prototype. Figure 3 illustrates the screenshot of the running demonstration.

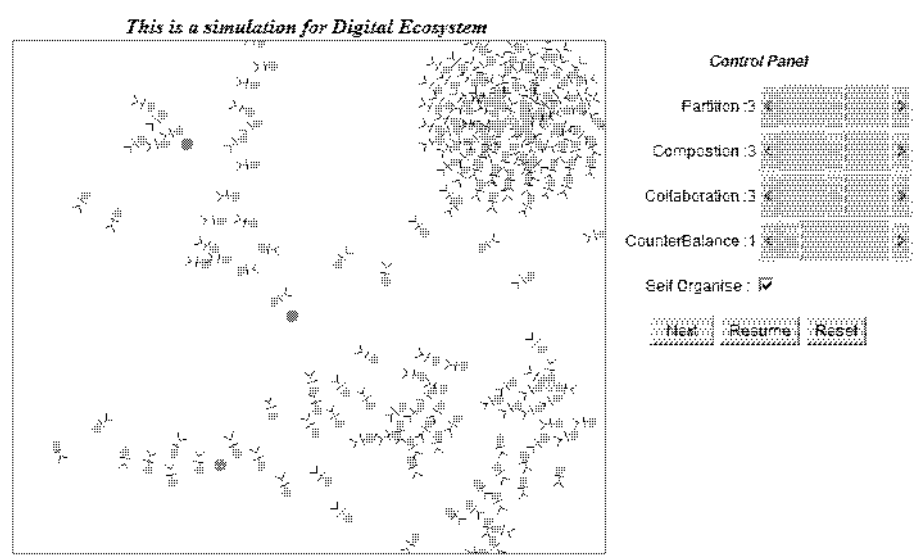

Figure 3. Screenshot of the Demo

The simulation user interface comprises two parts. On the left hand side, the simulation canvas presents the whole running environment where all species are rambling around guided by certain rules or strategies. Situating on the right, the control panel can be used to adjust the behaviour of each individual species, which in turn, affect the collective behaviour of other neighbouring species as a whole.

\section{A. Simulation Canvas}

Simulation Canvas, acting as the open environment, hosts the spaces of species. It can be regarded as the native environment of physical species, for example, a water pond full of various animals and botanies. In analogy to physical environment, the simulation canvas can also be considered as the "virtual environment" of digital species (e.g. intelligent agent), for example, the network infrastructure supporting inter-connected software applications, i.e. the World Wide Web.

Within the area of a canvas, we can see a great number of human-shaped "species" wondering around. These species live in the world of simulation canvas and interact with each other in order to survive and prosper. The seemingly random characteristics of their walking trails indicate that they attempt to find better location to obtain satisfactory opportunities within their space - the simulation canvas. However, their movement - the direction and the speed - is not absolutely random. They do follow several rules to interact and collaborate with each other, which enable them as a whole to quickly find and consume scarcely located opportunities - the coloured dots. In general, species tends to wonder around places where they can find more species "mates". Hence, their movements are heavily influenced by their neighbours" behaviour. This is caused by following a simple strategy "always learning from the average trend of your neighbours". Nevertheless, such a strategy can be overridden by applying other particular rules. We will explore the complete set of rules in the next section - Control Panel.

Another important entity in the canvas is the opportunity the coloured dots as indicated in the Figure 3. These opportunities attract different species. They can grow in terms of magnitude when more and more species have decided to stay and consume their offered resources. In particular, Figure 3 features five opportunities - two red ones, one pink one, and one green. Different colour represents different magnitude the number of species "hooked" in this very opportunity. Table 1 shows such a mapping between colours and numbers in our simulation setting.

Table 1. Mapping between Colours and Numbers

\begin{tabular}{|c|c|}
\hline Number of Species & Colour of Opportunities \\
\hline$<=10$ & Green \\
\hline$(10-20]$ & Pink \\
\hline$(20-40]$ & Orange \\
\hline$(40-50]$ & Magenta \\
\hline$>50$ & Red \\
\hline
\end{tabular}

As indicated Table 1, every opportunity will go through a 'coloured' life cycle - from green, to pink, to orange, to magenta, and eventually to red. When an opportunity turns red and the species still keep gathering in its outer circle, the opportunity might become "overheated" by too many attracted species. In this case, the opportunity will soon declare its resource is depleted as a result of being tapped by overwhelming species. On receiving the message 'overheat' or 'resource depletion', the surrounding species simply walk away or ignore this opportunity. Such a rule is driven by the nature of each specimen - seeking opportumity to survive and prosper. The two red dots in Figure 3 illustrate exactly this situation, where the resources are no longer attractive to those 
species, who just avoid the opportunities as though they were some kind of road barriers.

However, the opportunity can re-activate its own resource after a certain amount of time - a period called 'cooling' process. During the cooling process, the opportunity cools itself down by changing its colour in a reversed way - from red, to magenta, to orange, to pink, and eventually back to green. The magenta dot in Fig 3 (the bottom left one) illustrates such a cooling process, during which no species are interested in this opportunity unless it reaches its original status - the green colour as shown in the green dot (the bottom right one). We can see that the green dot opportunity just started to attract a couple of (less than ten according to Table 1) species. It could be a newly-created fresh green opportunity or an opportunity that has just cooled down from the 'overheated' status.

The orange dot opportunity in Fig 3 (the top right one) has attracted a large number of species around itself. Consequently, the species self-organise themselves into a cluster that takes full advantage of the useful resource provided by the opportunity 'orange'. Each specimen benefits from staying in this cluster. Meanwhile, the bigger the cluster, the more influential it could be to attract more and more neighbouring species heading towards the centre of the cluster as shown in this orange dot.

\section{B. Control Panel}

Control panel enables the user to interact with this simulation tools. At this stage of simulation, we believe such an interaction mainly deals with parameter setting. Hence, it provides five widgets to set the parameters and three buttons to help the user to gain better observation during the simulation.

The Partition scrollbar controls the extent to which how each individual specimen try to avoid other mates in terms of directions. It can be adjusted based on the scale ranging from 1 to 5 , each of which represents the magnitude that species steer to avoid crowding species mates. Figure 4 shows the effect of setting high value of partition - each species toddles on its own, and is not affected by other neighbouring mates.

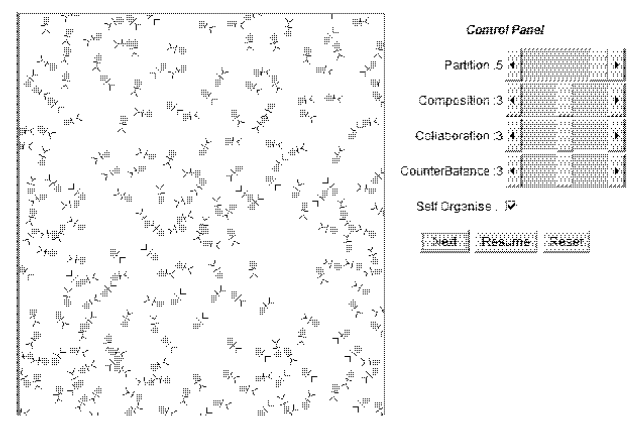

Figure 4 . When the partition is set high value

The Composition scrollbar determines to what degree that these species will gather together to form a cluster. Such a cluster is driven solely by this composition incentive rather than the opportunity discussed in the last section - Simulation
Canvas. Visually, it controls how these species will steer to move toward the average position of neighbouring species mates. Figure 5 depicts the outcome of placing high degree of composition - clusters are formed even without any stimuli or opportunities.

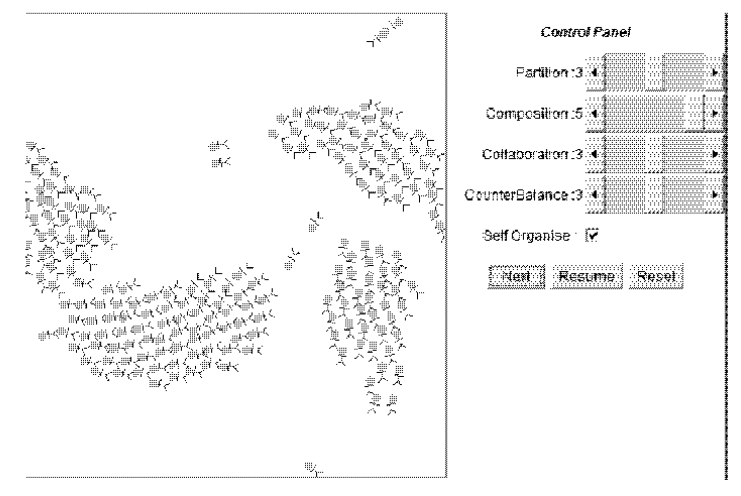

Figure 5. When the composition is put to high degree

The Collaboration scrollbar manages the level at which the species are willing to collaborate with each other to perform certain task. Visually, it stipulates how species steer towards the average heading of neighbouring species mates. Higher level of collaboration represents higher alignment of these species as shown in Figure 6.

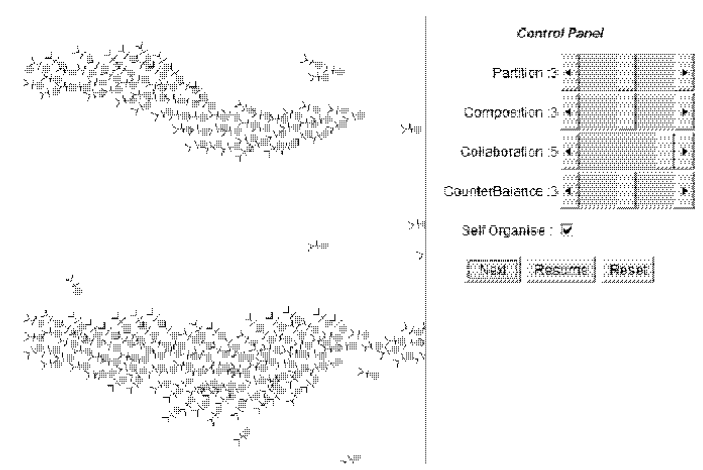

Figure 6 . When the collaboration is set to value of 5

The Counterbalance scrollbar is used to control the scale of each opportunity-driven cluster. The motivation is to set the threshold of the cluster size - i.e. to dismiss the cluster if the threshold is reached otherwise to keep attracting more species. In the reality, each type of opportunity has its own optimised threshold value - some opportunity needs $30-40$ species to cooperate, whereas others might only require $3-4$ species. In this simulation, we have not distinguished between different opportunities. We leave this to our future simulation work.

The Self-Organise check box is a switch to decide whether these species will form clusters around opportunities. By default, it is turned on so that opportunity-driven clusters are desired. However, there are some situations where we would like to observe the behaviour of species that are not attracted by opportunities. An appropriate real example could be the 
sensor networks, where each sensor (the dot opportunity in the simulation) just silently observes the species lurking and records their behaviours.

The pause/next and resume buttons are used by the user to gain better observation of the simulation. When the pause button is pressed, the simulation pauses and the user can have a 'snapshot' of the species - to further investigate how they are forming cluster, which directions they are walking, etc. The reset button is to help users quickly re-run random simulations repeatedly. We believe these controls will be more useful when being used together with some statistics data and graphs, which is one of our future goals for this simulation tools.

In this section, we have gone through the useful controls. From a broader perspective, the parameters set by these controls can be seen as species' survival rules. These rules can be "best practices" for recommending component, one of the core parts of digital ecosystems.

\section{Collaboration Style}

All the aforementioned system demonstrations are carried out under the default collaboration style - Swarm, where each specimen makes decisions on its own without giving in to some leaders or supervisors. This is adequate for the swarm intelligence research. However, during the research of digital ecosystems, we have found out alternative ways to collaborate. Hence, in the newer version of this simulation prototype, we have introduced the concepts of 'Collaboration Style' - the pattern stipulating how species are collaborated with each other. Besides the default Swarm style, we have thus explored the following collaboration styles.

Circle style. In this style, each specimen follows the previous specimen (while the first one following the last one) to form a circle just like a fish school. The motivation/reason for such a circle could be multi-folds: for instance, species are following one after another, but the first specimen somehow is attracted by the last specimen in the queue. A metaphor in reality would be the ring shape for the network topology. This is shown in the Figure 7

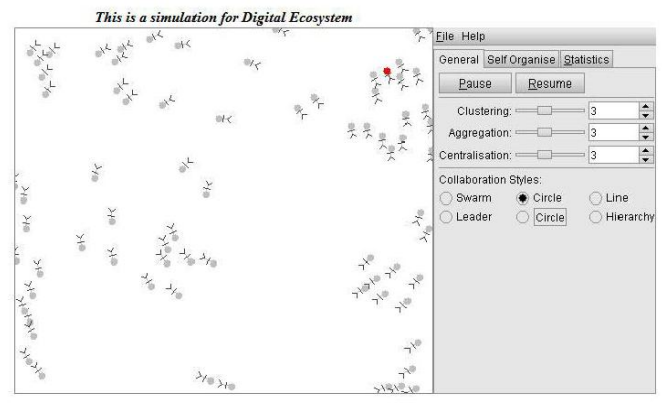

Figure 7. The circle style for DES

Line style. In this style as shown in Figure 8, a simple collaboration is carried out where only previous-next communication is conducted. Since it saves the frequency for communication, it would be useful for situations where only limited communication resources are available among species.

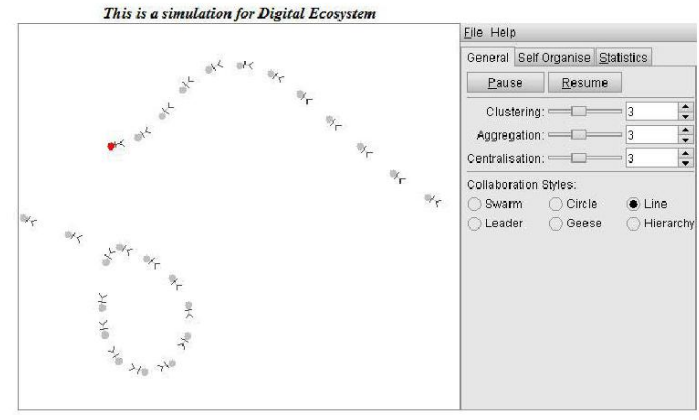

Figure 8. The line style for DES

Leader style. A leader is responsible for communicating among all other species. As depicted in the Figure 9, all other species follow leader's walking trail, thus learning from the leader to gain the best practices as a survival rule.

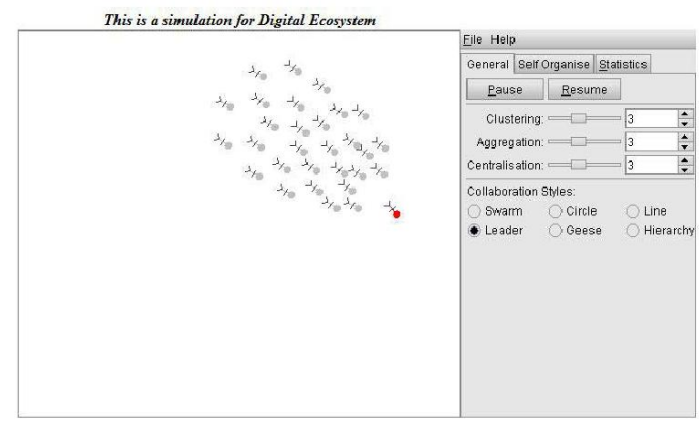

Figure 9. The leader style for DES

$V$-Shape. Just like a group of flying geese, the collaboration/communication can be visualised as a V-Shape as shown in Figure 10. The motivation for V-Shape lies in the physical ecosystem as well-known phenomena: geese take turn to become the leader, with other geese following the previous one, who can provide some extra air currency that can facilitate other followers flying more efficiently.

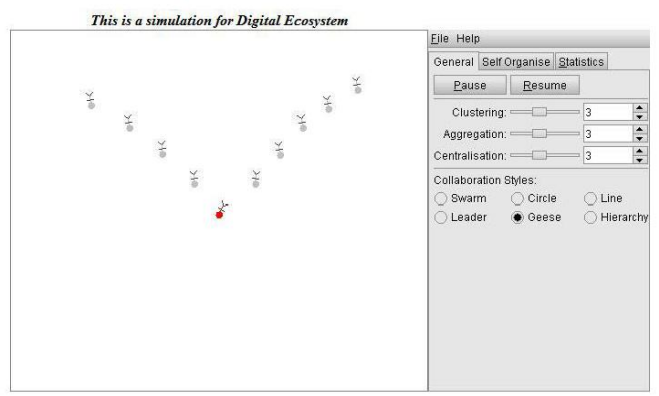

Figure 10. The V-Shape style for DES

Hierarchy Style. Like most organisations, a hierarchical structure is in place for collaboration management. In Figure 11, each specimen follows its immediate parent specimen, 
rather than the centralised leader. One specimen can have several children species under its administration.

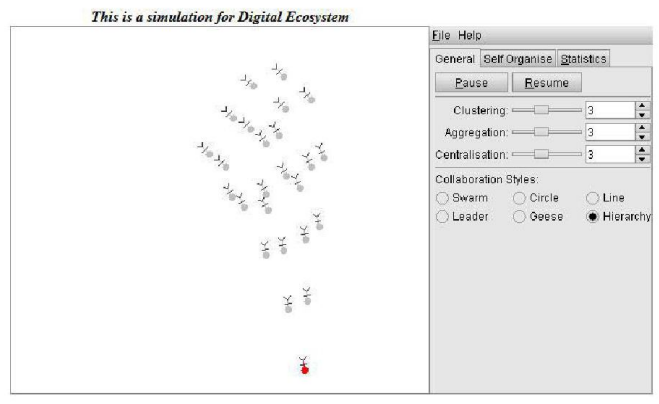

Figure 11. The hierarchy style for DES

\section{DES: A SOCIAL SOFTware REALISATION}

In this section, we provide a realisation of such a conceptual model and simulation prototype - Digital Ecosystem Collaboration platform. We have developed this wiki-based website to demonstrate all the concepts stated in the DES model and simulation. The Web UI of this platform is show in the Figure 12.

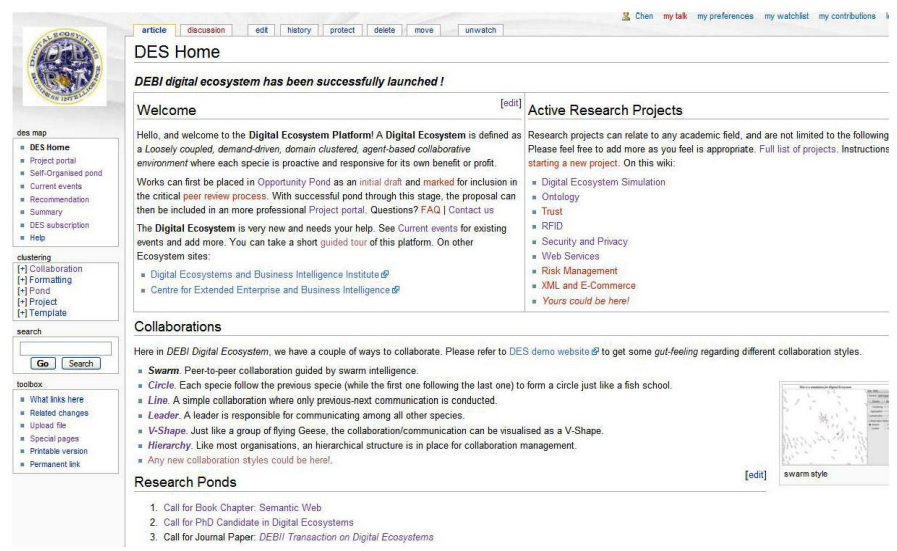

Figure 12. The screenshot for the DES collaboration platform

\section{A. User Interface Layout}

The collaboration platform is built on top of the MediaWiki, the underlying open source for Wikipedia. The rationale for choosing Wikis platform lies in the facts that free collaboration based on guided recommendation is the key for today's wiki prosperity, we believe this principle holds for the digital ecosystems, thus each specimen will collaborate with each other under some "best practice" (i.e. the counterpart for the "rule" defined in Section III) in response to some external stimuli - be it positive opportunity or negative signals. The concrete form of collaboration in our implementation is interpreted as "finishing some scientific research projects". The first front page consists of four major sections. Welcome section presents some basic rules for the user to participate in this DES collaboration platform. Since it is a part of Wiki section, the rules can also be edited -i.e. can be altered by any collaborators. It has the default (perhaps "best practice") rule set: any interesting topic shall firstly be placed into the research pond, after appropriate review process, it can then be introduced in the more formal research projects. Active Research Projects section presents existing mature research projects that are currently conducted by specifies - active researchers and scholars who participate and collaborate through this DES platform. Collaborations section presents all the collaboration styles that this platform supports. This is based on the styles described in Section IV DES simulation. Research Pond section lists all the potential opportunities (i.e. stimuli for species) that might attract some researchers who can work remotely through communicating via this DES collaboration platform. Once an opportunity becomes mature in the Research Pond, it will graduate from the incubator to the formal Active Research Projects section.

\section{B. Architectural Design}

The architecture design, as shown in figure 13, has four layers as a web-based application. End users can access this platform through multiple channels such as Web browsers, PDA via the standard HTTP communication protocol.

\begin{tabular}{|c|c|c|c|}
\hline User Layer & \multicolumn{3}{|c|}{ Web Clients (Browser, PDA, etc) } \\
\hline Network Layer & \multicolumn{3}{|c|}{ Apache Web Server (HTTP) } \\
\hline \multirow{4}{*}{ Logic Layer } & \multicolumn{3}{|c|}{ Digital Ecosystem Collaboration Platform } \\
\hline & \multicolumn{3}{|c|}{ MediaWiki's Extension Franework } \\
\hline & \multicolumn{3}{|c|}{ MediaWiki's PHP seripts } \\
\hline & \multicolumn{3}{|c|}{ PHP Engine } \\
\hline Data Layer & File Systems & MySQLDB & Caching System \\
\hline
\end{tabular}

Figure 13. The architecture for the DES collaboration platform

\section{CONCLUSIONS}

In this paper, we firstly introduce a DES conceptual model based on the Swarm research community. The implementation of a DES simulation prototype is then presented to illustrate some fundamental characteristics exhibited by DES. Finally, we propose a novel approach to realise the DES built on top of the Wikipedia Social Software. Currently, DES infrastructure architectural design is underway.

\section{REFERENCES}

E. Chang and M. West, "Digital Ecosystems and Comparison to Existing Collaboration Environment," WSEAS TRANSACTIONS on ENVRONMENT and DEVELOPMENT, vol. 2, pp. 1396 - 1404, 2006.

[2] "http://www.mediawiki.org/wiki/MediaWiki."

[3] "http://www.wikipedia.org/."

[4] "http://www.swarm.org/swarmdocs-2.2/refbook-java/." 